\title{
Drug-eluting stents or CABG?
}

Two studies published in the Journal of the American College of Cardiology and in the New England Journal of Medicine have compared drug-eluting stents with coronary artery bypass graft (CABG) for the treatment of either left main coronary artery disease (CAD) or multivessel CAD. "Previous trials may have been limited by their use of first-generation drug-eluting stents" explains Seung-Jung Park, who was an investigator in both studies. Both study reports include longterm follow-up data of second-generation drug-eluting stents that can help the selection of appropriate revascularization strategies.

The 1-year analysis of PRECOMBAT had previously shown that percutaneous coronary intervention (PCI) was noninferior to CABG for the primary end point of death, myocardial infarction (MI), stroke, or ischaemia-driven target-vessel revascularization. As a follow-up, Ahn et al. now report the 5-year results of PRECOMBAT, which randomly assigned 600 patients with unprotected left main coronary artery stenosis to PCI with a sirolimus-eluting stent or CABG. The primary end point of major adverse cardiac or cerebrovascular events (a composite of death from any cause, MI, stroke, or ischaemia-driven target-vessel revascularization) occurred at similar rates in both groups (cumulative event rates $17.5 \%$ vs $14.3 \%$; HR 1.27, $95 \%$ CI $0.84-1.90$; $P=0.26)$. Rates of death from any cause, MI, or stroke were also not different between the two groups, but the frequency of ischaemiadriven target-vessel revascularization was higher in the PCI group than in the CABG group (11.4\% vs 5.5\%; HR 2.11, 95\% CI 1.16-3.84; $P=0.012$ ). This trial lacked power to compare the composite safety end point of death, MI, or stroke; according to Park, we should wait for the results of the ongoing EXCEL trial (PCI with everolimus-eluting stent versus $C A B G$ ), which is adequately powered to assess death, MI, or stroke as a primary end point.

In the separate BEST study, researchers compared everolimuseluting stents to CABG in 880 patients with multivessel CAD. No differences in the primary end point of a composite of death, MI, or target-vessel revascularization were observed 2 years after the procedure (11.0\% vs $7.9 \%$; absolute risk difference $3.1,95 \%$ CI -0.8 to $6.9, P=0.32$ ). However, during a longer-term follow-up (median 4.6 years), the primary end point was more frequent in patients who received a stent than in those who underwent CABG (15.3\% vs $10.6 \%$; HR 1.47, 95\% CI 1.01-2.13, $P=0.04)$. In this extended period, a composite of death, MI, stroke, or repeat revascularization was also higher in the PCI group than in the CABG group (19.9\% vs $13.3 \%, P=0.01)$; this difference was probably the result of a higher rate of repeat revascularization in the PCI group than in the CABG group ( $11.0 \%$ vs $5.4 \%, P=0.003)$. On the basis of their findings, Park concludes that " $\mathrm{CABG}$ is still the preferred option for the treatment of multivessel disease".

These two studies suggest that both PCI and CABG have advantages and disadvantages depending on the clinical situation. "In the treatment of complex CAD, attending physicians should select the treatment option based on the scientific evidence and careful discussion with their patients," Park concludes.

João H. Duarte

Original articles Ahn, J. -M. et al. Randomized trial of stents versus bypass surgery for left main coronary artery disease: five-year outcomes of the PRECOMBAT study. J. Am. Coll. Cardiol. doi:10.1016/ j.jacc.2015.03.033. | Park, S. -J. et al. Trial of everolimus-eluting stents or bypass surgery for coronary disease. N. Engl. J. Med. doi:10.1056/NEJMoa1415447 